\title{
Overview of the outcomes of robotic segmentectomy and lobectomy
}

\author{
Giulia Veronesi $^{1,2}$, Pierluigi Novellis ${ }^{2}$, Gianluca Perroni ${ }^{3}$ \\ ${ }^{1}$ Faculty of Medicine and Surgery, Vita-Salute San Raffaele University, Milan, Italy; ${ }^{2}$ Division of Thoracic Surgery, IRCCS San Raffaele Scientific \\ Institute, Milan, Italy; ${ }^{3}$ Department of Thoracic Surgery, Humanitas Clinical and Research Center-IRCCS, Rozzano (Mi), Italy \\ Contributions: (I) Conception and design: G Veronesi; (II) Financial support: None; (III) Administrative support: G Veronesi; (IV) Provision of study \\ materials or patients: G Veronesi; (V) Collection and assembly of data: P Novellis; (VI) Data analysis and interpretation: G Perroni; (VII) Manuscript \\ writing: All authors; (VII) Final approval of manuscript: All authors. \\ Correspondence to: Giulia Veronesi. Thoracic Surgery Division, IRCCS Ospedale San Raffaele, Via Olgettina 60, 20132 Milan, Italy. \\ Email: veronesi.giulia@hsr.it.
}

\begin{abstract}
Segmentectomy has gained popularity in the latest years as a valid alternative to lobectomy. Initially reserved to patient unfit for lobar lung resection, this procedure is now offered also in selected patient with $<2 \mathrm{~cm}$ peripheral lung cancer confined to an anatomic segment with no nodal involvement on preoperative evaluation. The introduction of screening with low-dose CT chest scan allowed the identification of lung cancer at early stages, making possible to schedule a more conservative lung surgery. A major improvement came also from minimally invasive surgery (MIS), reducing complication rate with comparable survival rates when compared to open surgery. However, due to long learning curve and uncomfortable instruments handling of video-assisted thoracoscopy, many surgeons still prefer to perform segmentectomies through a thoracotomy and thus increasing perioperative morbidity and leading to postthoracotomy syndrome due to rib-spreading. Robotic assisted thoracic surgery (RATS) can avoid this throwback, combining the handling of open surgery with lesser invasiveness of thoracoscopy. Although literature has given strong evidences in favour of robotic lobectomies, data are still limited regarding segmentectomies performed with this technique. Moreover, no results are still available from the two ongoing randomized controlled trials comparing segmentectomy to lobectomy and so the latter represent the oncologically proper procedure for lung cancer along with lymph-node dissection. In this review we analyse the literature currently available on outcomes of lobar and sublobar anatomical resection performed by RATS, with a brief mention of the existing surgical techniques of port positioning and the costs of this procedure.
\end{abstract}

Keywords: Lung cancer; segmentectomy; robotic surgery; minimally invasive surgery (MIS)

Submitted May 05, 2020. Accepted for publication Jul 31, 2020.

doi: $10.21037 /$ jtd-20-1752

View this article at: http://dx.doi.org/10.21037/jtd-20-1752

\section{Introduction}

The introduction of minimally invasive surgery (MIS) in thoracic surgery has been a great step forward to increase quality of life (QOL) of patients and to minimize the trauma related to traditional open surgery (1). Retrospective series and systematic review demonstrated that VATS Lobectomy is superior to thoracotomy in terms of perioperative outcome for lung major resections. Also, few analysis demonstrated advantages in oncological outcome, probably related to the minor trauma and therefore the lesser immunosuppressive effect (2-4). More recently the randomized study by Bendixen and colleagues showed advantages in terms of post-operative QOL and reduced pain in subjects undergoing VATS versus open lung lobectomy (5). Despite this consistent evidence in favour of VATS, this approach remains not fully adopted for the 
difficulties and discomfort to operate with a suboptimal bidimensional view and rigid instrument (6). In the need to overcome technical limitations of manual MIS, but integrating the minimal trauma with the benefits of open surgery, robotic approach has been introduced around 2000 ' and rapidly diffused among different specialities all over the world. In Italy we observe an increase of $14 \%$ of robotic procedures every year (7). The success of robotic was related to many indisputable technical advantages: 3 dimensional view, precision of movements, alignment between surgeon eyes and target, surgeon's comfort and an empowerment of his/her capabilities that can control up to 4 tools concurrently by two hands. Advantages in terms of clinical benefits for patients treated with robotic approach versus others minimally invasive techniques is more difficult to demonstrate for standard lobectomies, but in the last few years two major changes have been observed in the panorama of lung surgery. First of all, worldwide lung cancer screening has unequivocally led to an increase in lung tumours diagnosed in the early stages (pT1a and b) (8). Many of these neoplastic forms are indolent and lobectomy associated to extended lymphadenectomy, that is still considered the standard in lung cancer treatment, starts to be seen as an overtreatment for lung cancer. The normal consequences are that lung-sparing surgery, such as segmentectomy, is becoming more popular in thoracic surgery $(9,10)$. Secondly, robotic thoracic surgery can be a feasible treatment for locally advanced tumours. The possibility of using a minimally invasive technique with lower surgical stress for the patient in IIIA stage tumours, or for central lesions in which vascular or bronchial sleeve resections are necessary, is expanding considerably (11-13). In this overview on robotic lung surgery we want to focus on the outcomes of robotic lobectomies and segmentectomies but also on the latest news of the robotic approach. We did not perform a systematic review, but we find the latest evidences in favour of robotic surgery.

\section{Technical aspect of robotic lung resection}

Despite the doubts and the absence of definitive evidence, we believe that the robotic procedure is the real revolution of the minimal invasive surgery today. Robotic approach for lung surgery is constantly growing in terms of diffusion and enough evidence has been collected on robotic thoracic surgery it will represent the minimally invasive procedure of the future. Indeed, the well known edges of robotic surgery -improved dexterity due to increased degrees of movement of the instruments, high-definition $3 \mathrm{D}$ view, lack of the fulcrum effect, and superior surgeon comfort, promoted the acceptance of robot in thoracic surgery. The surgeon controls "master" joysticks on the console and the motions are transmitted via robot-arms ("slave") to instruments. The instruments provided with 7 degrees of freedom reproduce the surgeon's movements, filtering out tremor. The "master" has foot pedals that allow the surgeon to replace the arms and electrify the instruments. Surgical equipe position and instrumentation is similar to conventional VATS, with patient under general anaesthesia and double lumen intubation in lateral decubitus. Hip are flexed to improve stability. Two main technique can be described, whether or not the presence of utility incision and Co2 insufflation. Robotic Assisted Lobectomy with four arms and four incisions (RAL-4) was first described by Park and Veronesi and use a $3 \mathrm{~cm}$ utility port at IV-V intercostal space (ICS) anteriorly along with a lower $30^{\circ}$ camera incision at VII-VIII ICS on the mid-axillary line. On the left side, video-port may be more lateral to avoid the heart. Lastly, 2 supplementary incisions are crafted at the VIII ICS on the tip of the scapula and in the auscultatory triangle (14). The completely portal robotic lobectomy with 3 arms (CPRL-3) was described by Dylewski and use a $0^{\circ}$ camera placed at V-VI ICS over the major pulmonary fissure along with 2 more ports on the same ICS. An additional incision is made at the end of the XI rib to introduce suction, stapler and lately be enlarged for specimen extraction. Cerfolio added a supplementary arm (CPRL-4) and placed all the arms along the VII ICS starting from the mid-axillary line to 2-3 centimetres to the spinous process. The supplementary port is positioned 2-3 ribs lower. Both those techniques use CO2 insufflation $(15,16)$. Those techniques can be applied also for segmentectomies, but in our experience addition of intravenous administration of Indocyanine green (ICG) after ligation of bronchus and vessels allow the clear distinction between target (grey) and non-target (bright green) segments (17). ICG was diluted at a concentration of $2.5 \mathrm{mg} / 10 \mathrm{~mL}$. A $6-8 \mathrm{~mL}$ bolus was injected into a peripheral vein catheter followed by saline solution. This technique can now be defined as the standard in robotic segmentectomy given its wide diffusion. Geraci and colleagues recently presented their casuistry on 245 consecutive segmentectomies achieved by nodule localization using indocyanine green both bronchoscopically and intravenously that made possible the identification of the intersegmental plan, thus achieving an $\mathrm{R} 0$ resection in $100 \%$ of cases (18). 


\section{Segmentectomy}

"Anatomic segmentectomy" means the resection of one or more bronchopulmonary segments with isolation and resection of segmental bronchus, artery and vein. In 1995 the results of a randomized trial comparing the oncologic outcome of patients with clinical stage-1 (pT1ab-c N0) lung cancer (NSCLC) treated with conventional lobectomy versus limited resection (segmentectomy or wedge resection) (19) established that pulmonary lobectomy was the gold standard for the radical surgical treatment of lung cancer and they remained undisputed for over 10 years. Okada and colleagues in 2006 compared some non-randomized studies and observed that sublobar resections were acceptable in comparison to lobectomies for NSCLCs with an average diameter less than $2 \mathrm{~cm}$ (pT1a-b N0), even in patients with no comorbidities (20). The rate of pulmonary lobectomies from 1998 to 2012 has reduced (21). Data comparing oncological outcomes of segmental resections with lobectomies are still limited. So far, in patients with a good PS, current guidelines confirm lobectomy as the gold standard treatment for surgical stage I NSCLC (grade 1B) (22). Two ongoing randomized trials will clarify if segmentectomy is comparable to lobectomy in terms of oncological results, and if it will be correlated to better lung function preservation $(23,24)$. Anatomic segmentectomy are considered by experienced thoracoscopic surgeons a good option to spare lung tissue. Nevertheless, robotic assisted surgery has been introduced to overcome the technical limitations of manual VATS. In 2012, our group described the first series of patients undergoing pulmonary segmentectomy in two centers $(25,26)$ and concluded that the technique was feasible, reproducible and correlated with no major bleeding. Toker and colleagues in 2014 confirmed previous data with his experience on 21 patients ( 15 affected by oncological pathology), who underwent robotic-assisted pulmonary segmentectomy. The author did not observe conversions to thoracotomy and concluded that the number of lymph nodes removed appeared "oncologically acceptable" for early-stage lung cancer (27). In 2016, Cerfolio reported a series of 100 robotic-assisted segmentectomies. Results were good: R0 resection was achieved in all patients and a $0 \%$ 30 - and 90-day mortalities was observed. Local-recurrence at 30 months was observed in $3.4 \%$ (28). A more recent paper comparing robotic assisted thoracic surgery (RATS) and VATS segmentectomies found no differences in terms of postoperative complications (e.g., air leaks), but robotic

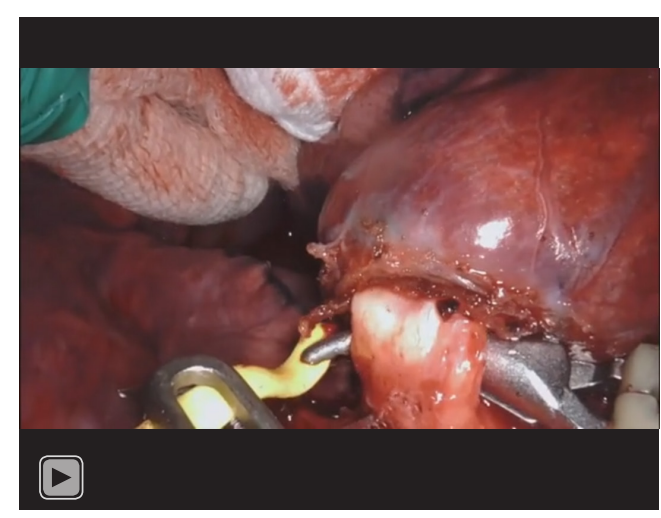

Video 1 Robotic right-S2 segmentectomy in a 59-years-old smoking woman, affected by a $14 \mathrm{~mm}$ PET-positive nodule of the right upper lobe, LDCT-screening detected.

approach performed better in lymph-node dissection with more nodes harvested in comparison to manual VATS (29). Casiraghi and colleagues published data from 10 years experience of RATS anatomical lung resections in 2018 performed on 339 patients, showing that 2- and 5-year overall survival (OS) was $96.2 \%$ for segmentectomies while $95.3 \%$ and $89.1 \%$ for lobectomies. Most of the cases where in clinical stage I and II. Limitations of this study are the retrospective data analysis and single center nature (30). The ongoing ROMAN study will further evaluate the differences in terms of conversion-to-open and postoperative complication rate between RATS and VATS, either lobectomy or segmentectomy (31). A video (Video 1) describes a robotic right S2 segmentectomy for a $14 \mathrm{~mm}$ pulmonary adenocarcinoma, p T1bN0, and underlines technical aspects and advantages.

\section{Lobectomy}

Minimally invasive approach is usually accepted for localized stage I or II lung cancers (32). A special mention has to be made regarding the technically challenging resectable tumors, in which the robotic approach could be advantageous. Although the use of VATS has been reported in patients with locally advanced NSCLC, few studies describe the use of the robotic approach (33-36). In our retrospective multicentric study on consecutive patients with clinically evident or occult N2 disease we observed that robotic approach is safe and effective in patients with Stage III NSCLC with a low conversion-rate and a low number of complications. Survival was similar to that reported for 
lobectomies performed with open thoracotomy (12). The benefit of the robotic approach over open thoracotomy is directly related to reduced surgical trauma and improved tolerability especially in fragile patients that received induction treatment. In the event of occult N2 disease, patients undergoing robotic lobectomies have a quicker recovery compared to those who receive open surgery and have an improved compliance with adjuvant treatments. Probably, in addition, a potential oncological benefit can be related to a lower immune response.

\section{Retrospective single center analysis}

Initial papers were focused on comparison of robotic versus open surgery. In 2010, we published the data of our first study regarding robotic and open lobectomies, focusing on the surgical outcomes of 54 robotic lobectomies versus 54 open lobectomies using a propensity score matching and assessing the learning curve of robotic lobectomy. Complications, postoperative stay and operative time declines after the first tertile of 18 robotic cases thus allowing to define as around this value the threshold for learning curve. Postoperative length of stay (LOS) was shorter after robotic compared to open procedures, while we demonstrated that complications and number of lymph nodes removed were comparable between the two techniques thus suggesting the safety and radicality of the robotic procedure (14). Cerfolio embraced robotic surgery in 2008, publishing early outcome of robotic and open lobectomies in 2011 (37). He demonstrates lower morbidity and mortality after robotic series with improved mental QOL and shorter hospital stay. He also described a similar N1 and N2 lymph node dissection and underlined how robotic allows " $\mathrm{R} 0$ resection for tumours up to $9.4 \mathrm{~cm}$ and outstanding mediastinal lymph node dissection (37). In a case control study, Louie and colleagues showed a significantly shorter duration of narcotic use and earlier return to normal activities after robotic approach compared to VATS for lung lobectomies, but similar operative outcome. According to the authors the advantage of the robotics approach is the greater confidence in dissecting N1 lymph nodes adjacent to the pulmonary artery and the easier and safer passage of the stapler (38). Kneuertz and colleagues compared nodal upstaging following lobectomy performed via open, RATS and VATS method for clinically N0/N1 NSCLC. They observed that, compared with a traditional thoracotomy approach, robotic lobectomy was associated with similar results. Nodal upstaging was lower when comparing VATS to thoracotomy, but no difference was found between the latter and RATS. A thorough evaluation of hilar and mediastinal LNs remains critical to ensure accurate staging by detection of occult LN metastases (39). The advantage of robotic surgery, however, is showing itself as not only technical. Kneuwertz and colleagues analyzed high risk patients with a reduced FEV1. Authors hypothesized that robotic lobectomy could have given some advantage in high-risk patients. A cohort of 599 patients undergone lobectomy by robotic $(n=287)$ or open $(\mathrm{n}=312)$ approach, including 189 high-risk patients was analyzed. Results shown that robotic lobectomy arm had a lower rate of prolonged air leak ( $6 \%$ vs. $10 \%$, $\mathrm{P}=0.047$ ), atelectasis requiring bronchoscopy ( $6 \%$ vs. $16 \%$, $\mathrm{P}=0.02)$, pneumonia ( $3 \%$ vs. $8 \%, \mathrm{P}=0.01$ ) and shorter LOS (4 vs. 6 days, $\mathrm{P}=0.001$ ). Overall pulmonary complication rate was significantly lower after robotic lobectomy in highrisk patients ( $28 \%$ vs. $45 \%, \mathrm{P}=0.02)$. Authors concluded that robotic lobectomy reduced the risk of postoperative pulmonary complication as compared with traditional open thoracotomy. In particular in patients with a limited pulmonary function robotic approach had the greatest benefit (40). However, it must be considered that, according to our recent paper, subjects with impaired respiratory function are those with highest risk of developing an intraoperative vascular complication that can require rapid conversion. They can also develop potentially more fatal respiratory complications (41-43).

\section{Multicentric studies review and metanalysis}

Some review paper and metanalysis compared VATS and robotic cases and found no significant differences in perioperative complications and mortality. Kent and colleagues analysed data from high-volume pulmonary surgeons (more than 20 lobectomies/year) and found that robotic lobectomies were associated with lower mortality rates ( $0 \%$ vs. $1.9 \%, \mathrm{P}=0.011$ ), LOS (5.9 vs. 7.4 days, $\mathrm{P}=0.015)$, and complication rate $(42.9 \%$ vs. $53.0 \%, \mathrm{P}=0.008)$ compared with open approach. Interestingly, among highvolume surgeons, robotic lobectomy was also associated with a statistically significant reduction of in-hospital mortality when compared with VATS lobectomy ( $0 \% v s$. $1.6 \%, \mathrm{P}=0.02$ ) (44). Similarly the metanalysis by Emmert in 2014, including 3,375 RVATS resection and 58,683 VATS, showed a benefit in term of mortality reduction with an HR of 0.52 in favour of robotic lobectomy and a trend of LOS and hospitalisation in reduction (45). 
The oncological adequacy has been shown by a large retrospective multicenter study that involved more than 300 cases in three different centers. Survival rates of patients treated with robotic lobectomy for early stage lung cancer were comparable to that of open and VATS approach (46). Reddy and colleagues compared results from a national database obtained by proficient surgeons who performed both robotic and VATS lobectomies. The authors included in their analysis 9,360 VATS lobectomies and 2,994 RATS lobectomies. Propensity-matched comparison showed that robotic-assisted procedures had a longer mean operative time by 25 minutes (mean 247.1 vs. 222.6 minutes, $\mathrm{P}<0.0001)$ but had a lower conversion rate $(4.8 \%$ vs. $8.0 \%$, $\mathrm{P}=0.007)$ and a lower 30 -day complication rate $(33.4 \%$ vs. 39.2\%, $\mathrm{P}=0.0128$ ) (47). Considering an established indicator of quality of care "readmission after surgery", Bailey and colleagues compared this indicator in patients underwent open, VATS and RATS lobectomies in the Nationwide Readmissions Database (NRD). The Authors observed a significantly lower readmission rates, better clinical outcomes and lower overall costs in the minimally invasive approach compared to open surgery. VATS and robotic surgery had similar readmission and mortality rates, however VATS was associated with a significantly reduced risk of short-term complications and lower cost (48). Oh and colleagues analysed perioperative clinical outcomes from robotic, VATS and open lobectomies. Data were acquired by the Premier Healthcare Database in a cohort of patients undergoing surgery from 2011 to 2015. The authors observed that robotic lobectomies were associated with a lower postoperative complication rate $(\mathrm{P}<0.0001)$, shorter hospital stay $(\mathrm{P}<0.0001)$, and lower mortality rate $(\mathrm{P}=0.0282)$. Patients in the robotic-assisted lobectomy (RL) group were more likely to be discharged home than to a transitional health care facility $(\mathrm{P}<0.0001)$. Compared with VATS, the robotic group had a lower conversion rate to thoracotomy $(\mathrm{P}<0.0001)$, lower overall postoperative complication rate $(\mathrm{P}=0.0061)$, and shorter hospital stay $(\mathrm{P}=0.006)$. The postoperative mortality rates of robotic and VATS were similar $(\mathrm{P}=0.44)$. The conclusions of the study confirmed that Robotic-assisted lobectomy was associated with improved outcomes for certain perioperative clinical variables, including shorter LOS and lower complication rates. It was also associated with a lower conversion rate to thoracotomy compared with VATS (49). Cerfolio and colleagues recently published a 4 institutions retrospective database review of RATS, finding excellent 5-year stage- specific survival for non-small cell lung cancer. This result was even better when compared to data from literature open surgery, hypotizing a reduction of immunosuppression state after operation (50). To answer ever more current questions in terms of lung surgery (practiced through the various techniques: open, multiportal VATS, single-port VATS and robotics) a recent ISMICS survey raised some questions (51). The results of this survey were that VATS is more advantageous than traditional surgery particularly with respect to adverse events, pain control and perhaps improved survival. Robotic approach may be more expensive than VATS and there is a suggestion that uniportal VATS may be associated with lower adverse events and pain (52). To validate any difference in terms of quality of surgery a prospective multinational randomised study is ongoing to compare early outcome of robotic assisted lobectomy and segmentectomy versus open.

\section{Cost analysis}

Higher procedural costs were considered the main limitation of the robotic approach. Oh and colleagues retrospectively analysed RATS and VATS procedures, showing higher costs (around 2.500 dollars) of the robotic procedures compared with the thoracoscopic approach with same LOS (53). However, some limitations should be underlined including that the majority of robotic centers were at the beginning of their learning curve, with only 8 cases performed. Subramanian and colleagues observed that minimally invasive approaches were associated to improved clinical outcomes compared with open lobectomy, but only robotic-assisted lobectomy has had rapid growth in utilization. One of the main reasons can be found in a shorter learning curve (20 vs. 50 cases). However, costs were still higher for RATS surgery when compared to thoracotomy (54). Different results were reached by Kneuertz et al.: they compare open lobectomy with MIS and founding that, although procedural costs was higher for RATS, overall costs were similar with $\$ 17,223$ (robot) vs. $\$ 17,260$ (VATS), vs. $\$ 18,075$ (open), $\mathrm{P}=0.48)$. Increased procedural cost of minimally invasive lobectomy can be recovered by postoperative costs reductions, associated with improved postoperative outcomes and shorter hospital stay (55). We compared the costs of the three different approaches in lobectomy. In a nation in which reimbursements are provided by a national healthcare system and based on Disease Related Group 
(DRG), the higher costs of robotic approach still allowed a profit margin of $18 \%$ (56). A retrospective analysis of 50 segmentectomies even found out a reduction in overall costs for this procedure ( $\$ 3650$ for RATS versus $\$ 4850$ for VATS) thank to a shorter LOS and the use of manual stapler instead of the more expensive robotic one (57).

\section{Conclusions}

Many steps forward have been made since robotic surgery was first introduced into clinical practice. Anatomic segmentectomy is increasing worldwide thanks to the spread of early diagnosis. It is precisely in segment surgery that the robotic approach finds its great clinical application. Besides, some evidences suggest advantages also in locally advanced neoplasms. This is mainly due to a faster functional recovery, therefore adjuvant treatments may be initiated earlier. However, more studies are needed to validate these findings. Initial cost and maintenance of robotic technology is still a limitation, although the availability in the future of robotic platforms from other suppliers besides Intuitive will lower prices, therefore making RATS more accessible and widespread.

\section{Acknowledgments}

Funding: None.

\section{Footnote}

Provenance and Peer Review: This article was commissioned by the Guest Editor (Ghulam Abbas) for the series "Robotic Thoracic Surgery" published in Fournal of Thoracic Disease. The article has undergone external peer review.

Conflicts of Interest: All authors have completed the ICMJE uniform disclosure form (available at: http://dx.doi. org/10.21037/jtd-20-1752). The series "Robotic Thoracic Surgery" was commissioned by the editorial office without any funding or sponsorship. GV serves an unpaid editorial board member of Fournal of Thoracic Disease from Jan 2020 to Dec 2021. GV reports grants from INAIL, during the conduct of the study; personal fees from Medtronic, personal fees from Ab Medica, personal fees from Johnson \& Johnson, grants from Intuitive, outside the submitted work. The authors have no other conflicts of interest to declare.
Ethical Statement: The authors are accountable for all aspects of the work in ensuring that questions related to the accuracy or integrity of any part of the work are appropriately investigated and resolved.

Open Access Statement: This is an Open Access article distributed in accordance with the Creative Commons Attribution-NonCommercial-NoDerivs 4.0 International License (CC BY-NC-ND 4.0), which permits the noncommercial replication and distribution of the article with the strict proviso that no changes or edits are made and the original work is properly cited (including links to both the formal publication through the relevant DOI and the license). See: https://creativecommons.org/licenses/by-nc-nd/4.0/.

\section{References}

1. Demmy TL, Nwogu C. Is video-assisted thoracic surgery lobectomy better? Quality of life considerations. Ann Thorac Surg 2008;85:S719-28.

2. Oda R, Okuda K, Osaga S, et al. Long-term outcomes of video-assisted thoracoscopic surgery lobectomy vs. thoracotomy lobectomy for stage IA non-small cell lung cancer. Surg Today 2019;49:369-77.

3. Whitson BA, D'Cunha J, Maddaus MA. Minimally invasive cancer surgery improves patient survival rates through less perioperative immunosuppression. Med Hypotheses 2007;68:1328-32.

4. Murakawa T, Ichinose J, Hino H, et al. Long-term outcomes of open and video-assisted thoracoscopic lung lobectomy for the treatment of early stage non-small cell lung cancer are similar: a propensity-matched study. World J Surg 2015;39:1084-91.

5. Bendixen M, Jørgensen OD, Jørgensen OD, et al. Postoperative pain and quality of life after lobectomy via video-assisted thoracoscopic surgery or anterolateral thoracotomy for early stage lung cancer: a randomised controlled trial. Lancet Oncol 2016;17:836-44.

6. Velez-Cubian FO, Ng EP, Fontaine JP, et al. RoboticAssisted Videothoracoscopic Surgery of the Lung. Cancer Control 2015;22:314-25.

7. Louie BE, Wilson JL, Kim S, et al. Comparison of VideoAssisted Thoracoscopic Surgery and Robotic Approaches for Clinical Stage I and Stage II Non-Small Cell Lung Cancer Using The Society of Thoracic Surgeons Database. Ann Thorac Surg 2016;102:917-24.

8. Veronesi G, Maisonneuve P, Rampinelli C, et al. 
Computed tomography screening for lung cancer: results of ten years of annual screening and validation of cosmos prediction model. Lung Cancer 2013;82:426-30.

9. Veronesi G, Maisonneuve P, Pelosi G, et al. Screeningdetected lung cancers: is systematic nodal dissection always essential? J Thorac Oncol 2011;6:525-30.

10. Mimae T, Okada M. Are segmentectomy and lobectomy comparable in terms of curative intent for early stage non-small cell lung cancer? Gen Thorac Cardiovasc Surg 2020;68:703-6.

11. Veronesi G, Park B, Cerfolio R, et al. Robotic resection of Stage III lung cancer: an international retrospective study. Eur J Cardiothorac Surg 2018;54:912-9.

12. Jiao W, Zhao Y, Qiu T, et al. Robotic Bronchial Sleeve Lobectomy for Central Lung Tumors: Technique and Outcome. Ann Thorac Surg 2019;108:211-8.

13. Pan X, Gu C, Wang R, et al. Initial Experience of Robotic Sleeve Resection for Lung Cancer Patients. Ann Thorac Surg 2016;102:1892-7.

14. Veronesi G, Galetta D, Maisonneuve P, et al. Four-arm robotic lobectomy for the treatment of early-stage lung cancer. J Thorac Cardiovasc Surg 2010;140:19-25.

15. Cerfolio RJ. Total port approach for robotic lobectomy. Thorac Surg Clin 2014;24:151-6.

16. Dylewski MR, Ohaeto AC, Pereira JF. Pulmonary resection using a total endoscopic robotic video-assisted approach. Semin Thorac Cardiovasc Surg 2011;23:36-42.

17. Pardolesi A, Veronesi G, Solli P, et al. Use of indocyanine green to facilitate intersegmental plane identification during robotic anatomic segmentectomy. J Thorac Cardiovasc Surg 2014;148:737-8.

18. Geraci TC, Ferrari-Light D, Kent A, et al. Technique, Outcomes With Navigational Bronchoscopy Using Indocyanine Green for Robotic Segmentectomy. Ann Thorac Surg 2019;108:363-9.

19. Ginsberg RJ, Rubinstein LV. Randomized trial of lobectomy versus limited resection for T1 N0 non-small cell lung cancer. Ann Thorac Surg 1995;60:615-22.

20. Okada M, Koike T, Higashiyama M, et al. Radical sublobar resection for small-sized non-small cell lung cancer: a multicenter study. J Thorac Cardiovasc Surg 2006;132:769-75.

21. McMurry TL, Shah PM, Samson P, et al. Treatment of stage I non-small cell lung cancer: What's trending?. J Thorac Cardiovasc Surg 2017;154:1080-7.

22. Howington JA, Blum MG, Chang AC, et al. Treatment of stage I and II non-small cell lung cancer: Diagnosis and management of lung cancer, 3rd ed: American College of Chest Physicians evidence-based clinical practice guidelines. Chest 2013;143:e278S-e313S.

23. Altorki NK, Wang X, Wigle D, et al. Perioperative mortality and morbidity after sublobar versus lobar resection for early-stage non-small-cell lung cancer: post-hoc analysis of an international, randomised, phase 3 trial (CALGB/Alliance 140503). Lancet Respir Med 2018;6:915-24.

24. Nakamura K, Saji H, Tsuboi M, et al. A Phase III Randomized Trial of Lobectomy Versus Limited Resection for Small-sized Peripheral Non-small Cell Lung Cancer (JCOG0802/WJOG4607L). Jpn J Clin Oncol 2010;40:271-4.

25. Pardolesi A, Veronesi G. Robot-assisted Lung Anatomic Segmentectomy. Thorac Surg Clin 2014;24:163-8.

26. Pardolesi A, Park B, Petrella F, et al. Robotic anatomic segmentectomy of the lung: technical aspects and initial results. Ann Thorac Surg 2012;94:929-34.

27. Toker A, Ayalp K, Uyumaz E, et al. Robotic lung segmentectomy for malignant and benign lesions. J Thorac Dis 2014;6:937-42.

28. Cerfolio RJ, Watson C, Minnich DJ, et al. One Hundred Planned Robotic Segmentectomies: Early Results, Technical Details, and Preferred Port Placement. Ann Thorac Surg 2016;101:1089-95.

29. Xie B, Sui T, Qin Y, et al. Comparison of Short-term Outcomes of Lung Segmentectomy by Robotic-assisted and Video-assisted Thoracoscopic Surgery. Zhongguo Fei Ai Za Zhi 2019;22:767-71.

30. Casiraghi M, Galetta D, Borri A, et al. Ten Years' Experience in Robotic-Assisted Thoracic Surgery for Early Stage Lung Cancer. Thorac Cardiovasc Surg 2019;67:564-72.

31. Videothoracoscopic (VATS) vs. Robotic Approach for Lobectomy or Anatomical Segmentectomy (ROMAN). ClinicalTrials.gov Identifier: NCT02804893.

32. Yan TD, Cao C, D'Amico TA, et al. Video-assisted thoracoscopic surgery lobectomy at 20 years: a consensus statement. Eur J Cardiothorac Surg 2014;45:633-9.

33. Huang J, Xu X, Chen H, et al. Feasibility of complete video-assisted thoracoscopic surgery following neoadjuvant therapy for locally advanced non-small cell lung cancer. J Thorac Dis 2013;5 Suppl 3:S267-73.

34. Nakanishi R, Fujino Y, Yamashita T, et al. Thoracoscopic anatomic pulmonary resection for locally advanced nonsmall cell lung cancer. Ann Thorac Surg 2014;97:980-5.

35. Hennon M, Sahai RK, Yendamuri S, et al. Safety of thoracoscopic lobectomy in locally advanced lung cancer. 
Ann Surg Oncol 2011;18:3732-6.

36. Gonzalez-Rivas D, Fieira E, Delgado M, et al. Is uniportal thoracoscopic surgery a feasible approach for advanced stages of non-small cell lung cancer? J Thorac Dis 2014;6:641-8.

37. Cerfolio RJ, Bryant AS, Skylizard L, et al. Initial consecutive experience of completely portal robotic pulmonary resection with 4 arms. J Thorac Cardiovasc Surg 2011;142:740-6.

38. Louie BE, Farivar AS, Aye RW, et al. Early experience with robotic lung resection results in similar operative outcomes and morbidity when compared with matched video-assisted thoracoscopic surgery cases. Ann Thorac Surg 2012;93:1598-604.

39. Kneuertz PJ, Cheufou DH, D'Souza DM, et al. Propensity-score adjusted comparison of pathologic nodal upstaging by robotic, video-assisted thoracoscopic, and open lobectomy for non-small cell lung cancer. J Thorac Cardiovasc Surg 2019;158:1457-1466.e2.

40. Kneuertz PJ, D'Souza DM, Moffatt-Bruce SD, et al. Robotic lobectomy has the greatest benefit in patients with marginal pulmonary function. J Cardiothorac Surg 2018;13:56.

41. Cao C, Cerfolio RJ, Louie BE, et al. Incidence, Management, and Outcomes of Intraoperative Catastrophes During Robotic Pulmonary Resection. Ann Thorac Surg 2019;108:1498-504.

42. Cao C, Louie BE, Melfi F, et al. Impact of pulmonary function on pulmonary complications after roboticassisted thoracoscopic lobectomy. Eur J Cardiothorac Surg 2020;57:338-42.

43. Cao C, Louie BE, Melfi F, et al. Outcomes of major complications after robotic anatomic pulmonary resection. J Thorac Cardiovasc Surg 2019:S0022-5223(19)31809-4.

44. Kent M, Wang T, Whyte R, et al. Open, video-assisted thoracic surgery, and robotic lobectomy: review of a national database. Ann Thorac Surg 2014;97:236-242; discussion 242-4.

45. Emmert A, Straube C, Buentzel J, et al. Robotic versus thoracoscopic lung resection: A systematic review and meta-analysis. Medicine (Baltimore) 2017;96:e7633.

46. Park BJ, Melfi F, Mussi A, et al. Robotic lobectomy for non-small cell lung cancer (NSCLC): Longterm oncologic results. J Thorac Cardiovasc Surg 2012;143:383-9.

47. Reddy RM, Gorrepati ML, Oh DS, et al. RoboticAssisted Versus Thoracoscopic Lobectomy Outcomes From High-Volume Thoracic Surgeons. Ann Thorac Surg
2018;106:902-8.

48. Bailey KL, Merchant N, Seo YJ, et al. Short-Term Readmissions After Open, Thoracoscopic, and Robotic Lobectomy for Lung Cancer Based on the Nationwide Readmissions Database. World J Surg 2019;43:1377-84.

49. Oh DS, Reddy RM, Gorrepati ML, et al. Robotic-Assisted, Video-Assisted Thoracoscopic and Open Lobectomy: Propensity-Matched Analysis of Recent Premier Data. Ann Thorac Surg 2017;104:1733-40.

50. Cerfolio RJ, Ghanim AF, Dylewski M, et al. The longterm survival of robotic lobectomy for non-small cell lung cancer: A multi-institutional study. J Thorac Cardiov Surg 2018;155:778-86.

51. Ng CSH, MacDonald JK, Gilbert S, et al. Expert Consensus Statement on Optimal Approach to Lobectomy for NonSmall Cell Lung Cancer. Innovations (Phila) 2019;14:87-9.

52. Ng CSH, MacDonald JK, Gilbert S, et al. Optimal Approach to Lobectomy for Non-Small Cell Lung Cancer: Systemic Review and Meta-Analysis. Innovations (Phila) 2019;14:90-116.

53. Swanson SJ, Miller DL, McKenna RJ, et al. Comparing robot-assisted thoracic surgical lobectomy with conventional video-assisted thoracic surgical lobectomy and wedge resection: results from a multihospital database. J Thorac Cardiovasc Surg 2014;147:929-37.

54. Subramanian MP, Liu J, Chapman WC, et al. Utilization Trends, Outcomes, and Cost in Minimally Invasive Lobectomy. Ann Thorac Surg 2019;108:1648-55.

55. Kneuertz PJ, Singer E, D'Souza DM, et al. Hospital cost and clinical effectiveness of robotic-assisted versus videoassisted thoracoscopic and open lobectomy: A propensity score-weighted comparison. J Thorac Cardiovasc Surg 2019;157:2018-2026.e2.

56. Novellis P, Bottoni E, Voulaz E, et al. Robotic surgery, video-assisted thoracic surgery, and open surgery for early stage lung cancer: comparison of costs and outcomes at a single institute. J Thorac Dis 2018;10:790-8.

57. Musgrove KA, Hayanga JA, Holmes SD, et al. Robotic versus Video-Assisted Thoracoscopic Surgery Pulmonary Segmentectomy: A Cost Analysis. Innovations 2018;13:338-43.

Cite this article as: Veronesi G, Novellis P, Perroni G. Overview of the outcomes of robotic segmentectomy and lobectomy. J Thorac Dis 2021;13(10):6155-6162. doi: 10.21037/ jtd-20-1752 